Quim. Nova, Vol. 35, No. 5, 932-938, 2012

\title{
DEGRADAÇÃO DE CORANTES TÊXTEIS E REMEDIAÇÃO DE RESÍDUOS DE TINGIMENTO POR PROCESSOS FENTON, FOTO-FENTON E ELETRO-FENTON
}

\author{
Terezinha Salvador, Luiz H. Marcolino Jr. e Patricio Peralta-Zamora* \\ Departamento de Química, Universidade Federal do Paraná, CP 19081, 81531-990 Curitiba - PR, Brasil
}

Recebido em 28/6/11; aceito em 24/11/11; publicado na web em 23/1/12

\begin{abstract}
DEGRADATION OF TEXTILE DYES AND REMEDIATION OF DYING RESIDUES BY FENTON, PHOTO-FENTON AND ELECTRO-FENTON PROCESSES. In this work the degradation of textile dyes were evaluated, using Fenton, photo-Fenton and electro-Fenton processes. Under optimized conditions Fenton and photo-Fenton processes showed high decolorization capacity of the model dyes. The electro-Fenton process was carried out in an undivided electrochemical reactor (1000 $\mathrm{mL})$ equipped with a carbon-felt cathode $\left(253 \mathrm{~cm}^{2}\right)$ and a platinum gauze anode $\left(6 \mathrm{~cm}^{2}\right)$. Under optimal conditions $\left(\mathrm{J}: 1.6 \mathrm{~mA} \mathrm{~cm}{ }^{-2}, \mathrm{Na}_{2} \mathrm{SO}_{4}: 0.075 \mathrm{~mol} \mathrm{~L}^{-1}\right.$, pH: 3) $\mathrm{H}_{2} \mathrm{O}_{2}$ concentration of about $60 \mathrm{mg} \mathrm{L}^{-1}$ was observed. The addition of $\mathrm{Fe}^{2+}\left(15 \mathrm{mg} \mathrm{L}^{-1}\right)$ induces Fenton reactions that permit almost total decolorization of textile dyes.
\end{abstract}

Keywords: textile dyes; degradation; electro-Fenton.

\section{INTRODUÇÃO}

Nas últimas décadas, o fenômeno da contaminação ambiental alcançou proporções globais, principalmente em função da continua emissão de inúmeros resíduos domésticos e industriais, os quais, quando não adequadamente tratados, apresentam um elevado potencial poluente.

Dentro do contexto da atividade industrial é possível destacar operações de tingimento de fibras têxteis, processo este que é responsável pela geração de grandes volumes de resíduos líquidos, contendo elevada carga orgânica e forte coloração. ${ }^{1}$ Nos últimos anos, inúmeros esforços têm sido dedicados à proposta de sistemas de tratamento que permitam a remoção de corantes têxteis, incluindo sistemas de nanofiltração, ${ }^{2}$ processos de adsorção com sorbentes de diversa natureza, ${ }^{3,4}$ sistemas biológicos fundamentados no uso de bactérias, ${ }^{5}$ fungos ${ }^{6}$ e enzimas ${ }^{7}$ e, mais recentemente, sistemas de tratamento fundamentados em processos oxidativos avançados, como fotocatálise heterogênea ${ }^{8}$ e processos Fenton. ${ }^{9}$

Em função da usual resistência dos corantes têxteis frente a processos de natureza biológica e do caráter não destrutivo de muitas propostas de natureza física, os processos oxidativos avançados têm ganhado bastante destaque nos últimos anos, principalmente em razão da sua efetiva capacidade de degradação e mineralização de substratos sabidamente resistentes, incluindo corantes têxteis. ${ }^{10}$

Dentro deste contexto, deve ser dado destaque aos processos do tipo Fenton, os quais têm se mostrado bastante promissores na degradação de corantes têxteis, basicamente em função da sua elevada eficiência de degradação e relativa simplicidade operacional. ${ }^{11}$ Os principais fundamentos deste tipo de processo, assim como as suas principais vantagens e desvantagens, podem ser consultados em revisões nacionais e internacionais publicadas recentemente..$^{12,13}$

Dentre as principais dificuldades encontradas na aplicação de sistemas Fenton em grande escala é possível destacar o caráter crítico da concentração de peróxido de hidrogênio. Nos processos foto-Fenton (Equações 1-3), assistidos por radiação ultravioleta, visível ou solar, o processo de degradação se processa rapidamente, em função da geração de elevadas quantidades de radical hidroxila. Entretanto, a cinética da reação se torna rapidamente desfavorável, em razão do

*e-mail: zamora@ufpr.br rápido consumo de peróxido de hidrogênio. Infelizmente, a adição de elevadas concentrações iniciais de peróxido também se mostra desfavorável, em função da ação sequestrante de radical hidroxila exercida pelo excesso de peróxido, o que leva à geração de radical hidroperoxila, de menor poder oxidante (Equação 4).

$$
\begin{gathered}
\mathrm{Fe}^{2+}+\mathrm{H}_{2} \mathrm{O}_{2} \rightarrow \mathrm{Fe}^{3+}+\mathrm{HO}^{-}+\mathrm{HO}^{\bullet} \\
\mathrm{Fe}^{3+}+\mathrm{H}_{2} \mathrm{O}+\mathrm{h} v \rightarrow \mathrm{Fe}^{2+}+\mathrm{H}^{+}+\mathrm{HO}^{\circ} \\
\mathrm{H}_{2} \mathrm{O}_{2}+\mathrm{h} v \rightarrow 2 \mathrm{HO}^{\bullet} \\
\mathrm{HO}^{\bullet}+\mathrm{H}_{2} \mathrm{O}_{2} \rightarrow \mathrm{H}_{2} \mathrm{O}+\mathrm{HO}_{2}^{*}
\end{gathered}
$$

Para contornar este tipo de inconveniente, tem sido proposta a geração eletroquímica de peróxido de hidrogênio, principalmente por oxidação catódica de oxigênio (Equação 5) em eletrodos de difusão gasosa de natureza carbonácea. ${ }^{14-17}$

$$
\mathrm{O}_{2}+2 \mathrm{e}^{-}+2 \mathrm{H}^{+} \rightarrow \mathrm{H}_{2} \mathrm{O}_{2} \quad\left(\mathrm{E}^{\mathrm{o}}=0,69 \mathrm{~V}, \mathrm{NHE}\right)
$$

Neste trabalho avaliou-se a capacidade de sistemas eletro-Fenton, operados com cátodos de carbono-felt, em relação à degradação de corantes e à remediação de resíduos de tingimento. Para comparação, processos Fenton convencionais foram também estudados.

A utilização de produtos carbonáceos, como o carbono-felt (CF), é bastante descrita na literatura para a construção de eletrodos orientados à produção de $\mathrm{H}_{2} \mathrm{O}_{2} \cdot{ }^{18,19} \mathrm{Em}$ geral, estima-se que o uso de carbono-felt possibilita reações eletroquímicas efetivas, devido à particularidade de serem materiais tridimensionais porosos. Estes materiais providenciam áreas eletronicamente ativas superiores aos eletrodos planos, sendo esta uma característica muito importante nas reações que envolvem gases dissolvidos em água. O gás move-se pela estrutura porosa, dissolvendo-se e difundindo-se a partir do eletrólito, reagindo finalmente na interface trifásica (fases sólida, eletrólito e gasosa). ${ }^{20}$

\section{PARTE EXPERIMENTAL}

\section{Reagentes}

Os corantes reativos Azul QR 19 (CI) e Laranja Reativo 16 (CI), ambos da Aldrich, foram utilizados em solução aquosa em concentração de $20 \mathrm{mg} \mathrm{L}^{-1}$. 
Sulfato ferroso PA $\left(\mathrm{FeSO}_{4} \cdot 7 \mathrm{H}_{2} \mathrm{O}\right.$, ISOFAR) e peróxido de hidrogênio (Peróxidos do Brasil Ltda., 50\%, m/V) foram utilizados em solução aquosa. Quando necessário, a sua concentração foi determinada por espectrofotometria e titulação permanganométrica, respectivamente.

Oxigênio comercial foi White Martins, enquanto que outros reagentes utilizados foram de grau analítico.

\section{Processos Fenton e foto-Fenton}

Todos os experimentos foram realizados em um reator fotoquímico de bancada de $250 \mathrm{~mL}$ de capacidade, equipado com agitação magnética e refrigeração por água. A radiação foi fornecida por uma lâmpada a vapor de mercúrio de $125 \mathrm{~W}$, sem o bulbo protetor, inserida na solução com a proteção de um bulbo de vidro Pyrex.

Nos estudos de degradação foram utilizadas soluções aquosas de corante Azul QR-19 e Alaranjado 16 (200 mL, $\left.20 \mathrm{mg} \mathrm{L}^{-1}\right)$ em pH 3. $\mathrm{O} \mathrm{Fe}^{2+}$ foi utilizado em concentrações entre 5 e $15 \mathrm{mg} \mathrm{L}^{-1}$, enquanto que a concentração de $\mathrm{H}_{2} \mathrm{O}_{2}$ variou entre 50 e $150 \mathrm{mg} \mathrm{L}^{-1}$.

Durante os processos de degradação, alíquotas de $10 \mathrm{~mL}$ foram retiradas em intervalos regulares, filtradas em membrana de acetato de celulose $(0,45 \mu \mathrm{m})$ e submetidas a controle analítico.

\section{Processos eletro-Fenton}

Todos os experimentos preliminares foram conduzidos em uma cela aberta não dividida com capacidade de $500 \mathrm{~mL}$, equipada com agitação magnética e sistema de borbulhamento de oxigênio. Como cátodos foram utilizados placas de carbono-felt (gentilmente cedidas por Seecil Carbon Technologies Ltda), aço inox e Ti/ $/ \mathrm{TiO}_{2} \mathrm{RuO}_{2}$ $\left(\mathrm{DSA}^{\circledR}\right)$, todos com área superficial de $21 \mathrm{~cm}^{2}$, enquanto que como ânodos foram utilizados fios de platina $\left(0,7\right.$ e $\left.6,0 \mathrm{~cm}^{2}\right)$ e placas de aço inox e $\mathrm{Ti} / \mathrm{TiO}_{2} \mathrm{RuO}_{2}\left(\mathrm{DSA}^{\circledR}\right)$.

Em etapas posteriores, os processos eletro-Fenton foram aplicados em cela aberta não dividida com capacidade de $1000 \mathrm{~mL}$, equipada com agitação magnética e sistema de borbulhamento de oxigênio. Como cátodo foi utilizado um cilindro de carbono-felt de $253 \mathrm{~cm}^{2}$, enquanto que como ânodos foram utilizados fios de platina e placas de aço inox e $\mathrm{Ti} / \mathrm{TiO}_{2} \mathrm{RuO}_{2}\left(\mathrm{DSA}^{\circledR}\right)$.

Em todos os casos, a corrente foi fornecida por uma fonte externa (EMG 18134), em densidades que variaram entre 0,4 a $27 \mathrm{~mA} \mathrm{~cm}{ }^{-2}$. A diferença de potencial foi aferida utilizando-se um multímetro digital, marca Minipa ET-2110, e eletrodo de referência de $\mathrm{Ag} / \mathrm{AgCl}$ ( $\mathrm{KCl} 3 \mathrm{~mol} \mathrm{~L}^{-1}$ ).

Os ensaios foram realizados em $\mathrm{pH} 3$ e na presença do eletrólito $\mathrm{Na}_{2} \mathrm{SO}_{4}$, em concentrações entre 0,025 e $0,075 \mathrm{~mol} \mathrm{~L}^{-1}$.

\section{Controle analítico}

A eficiência dos processos em estudo foi avaliada através do controle dos seguintes parâmetros experimentais: espectroscopia eletrônica, produção e consumo de $\mathrm{H}_{2} \mathrm{O}_{2}$ e Ferro (II) e Fe (III).

\section{Espectroscopia eletrônica}

O acompanhamento do perfil espectrofotométrico das amostras foi realizado em espectrofotômetro Shimadzu UV-2401, monitorando-se a região compreendida entre 200 e $800 \mathrm{~nm}$. Todas as medidas foram realizadas em cubetas de quartzo de $1 \mathrm{~cm}$ de caminho óptico.

\section{Produção e consumo de $\mathrm{H}_{2} \mathrm{O}_{2}$}

A concentração de peróxido de hidrogênio foi avaliada espectrometricamente, utilizando-se uma metodologia modificada a partir de procedimentos descritos na literatura. ${ }^{21}$ Neste procedimento, peróxido de hidrogênio reage com vanadato de amônio, o que leva à formação do cátion peroxovanádio que absorve fortemente em $446 \mathrm{~nm}$. Foram elaboradas curvas analíticas com padrão de peróxido de hidrogênio, na faixa entre 2,5 e $100 \mathrm{mg} \mathrm{L}^{-1}$. O coeficiente de variação estabelecido para as análises foi de $1 \%$, enquanto que o limite de quantificação foi de $2,5 \mathrm{mg} \mathrm{L}^{-1}$.

\section{Ferro (II) e Fe (III)}

As determinações de $\mathrm{Fe}^{2+}$ e $\mathrm{Fe}^{3+}$ foram realizadas via espectroscopia UV-vis, utilizando-se metodologia fundamentada na reação de complexação entre $\mathrm{Fe}^{2+}$ e o-fenantrolina, conforme metodologia padrão da APHA. ${ }^{22} \mathrm{O}$ teor de $\mathrm{Fe}^{2+}$ é determinado diretamente, enquanto que a concentração de $\mathrm{Fe}^{3+}$ é avaliada após redução com hidroquinona. Em ambos os casos, íons ferrosos reagem com o-fenantrolina formando um composto intensamente colorido, que pode ser medido por espectrofotometria na região do visível $(508 \mathrm{~nm})$. As concentrações foram determinadas a partir de uma curva padrão, elaborada com sulfato ferroso amoniacal $\left[\mathrm{Fe}\left(\mathrm{NH}_{4}\right)_{2}\left(\mathrm{SO}_{4}\right)_{2} \cdot 6 \mathrm{H}_{2} \mathrm{O}\right]$.

\section{RESULTADOS E DISCUSSÃO}

\section{Degradação de corantes modelos pelo sistema Fenton}

Inicialmente, estudos de degradação do corante azul QR 19 foram realizados pelo processo Fenton convencional, principalmente objetivando-se a obtenção de resultados que permitissem uma avaliação comparativa dos diversos sistemas de tratamento aplicados ao longo do trabalho. O efeito de variáveis experimentais de relevância na capacidade de degradação de processos Fenton convencionais foi preliminarmente investigado, utilizando-se um sistema de planejamento fatorial de experimentos. As variáveis estudadas foram concentração de $\mathrm{H}_{2} \mathrm{O}_{2}$ e de $\mathrm{Fe}^{2+}$, cada uma das quais foi avaliada em dois níveis, acrescidos de um ponto central ensaiado em triplicata (Tabela 1). Como respostas foram utilizados parâmetros cinéticos do processo de descoloração (constante de velocidade e tempo de

Tabela 1. Condições experimentais e parâmetros cinéticos do processo de descoloração do corante azul QR 19 por processo Fenton (corante: $20 \mathrm{mg} \mathrm{L}^{-1}$; volume: $200 \mathrm{~mL}$; pH: 3)

\begin{tabular}{|c|c|c|c|c|}
\hline \multirow{2}{*}{ Variáveis } & \multicolumn{4}{|c|}{ Níveis } \\
\hline & \multicolumn{2}{|c|}{$(-)$} & 0 & $(+)$ \\
\hline $\mathrm{H}_{2} \mathrm{O}_{2}\left(\mathrm{mg} \mathrm{L}^{-1}\right)$ & \multicolumn{2}{|c|}{50} & 100 & 150 \\
\hline $\mathrm{Fe}^{2+}\left(\mathrm{mg} \mathrm{L}^{-1}\right)$ & \multicolumn{2}{|c|}{5} & 10 & 15 \\
\hline Experimento & $\mathrm{H}_{2} \mathrm{O}_{2}$ & $\mathrm{Fe}^{2+}$ & $\mathrm{k}_{\mathrm{a}}\left(\min ^{-1}\right)$ & $\mathrm{t}_{1 / 2}(\min )$ \\
\hline 1 & - & - & 0,057 & 12,12 \\
\hline 2 & + & - & 0,104 & 6,69 \\
\hline 3 & - & + & 0,023 & 9,99 \\
\hline 4 & + & + & 0,422 & 1,64 \\
\hline 5 & 0 & 0 & 0,103 & 6,75 \\
\hline 6 & 0 & 0 & 0,112 & 6,19 \\
\hline 7 & 0 & 0 & 0,104 & 6,69 \\
\hline \multicolumn{5}{|c|}{ Efeitos calculados* } \\
\hline \multicolumn{3}{|c|}{ Conc. $\mathrm{H}_{2} \mathrm{O}_{2}$} & \multicolumn{2}{|l|}{0,223} \\
\hline \multicolumn{3}{|c|}{ Conc. $\mathrm{Fe}^{2+}$} & \multicolumn{2}{|l|}{0,142} \\
\hline \multicolumn{3}{|c|}{$\mathrm{H}_{2} \mathrm{O}_{2} \times \mathrm{Fe}^{2+}$} & \multicolumn{2}{|l|}{0,176} \\
\hline \multicolumn{3}{|c|}{ Desvio experimental } & \multicolumn{2}{|l|}{0,005} \\
\hline
\end{tabular}

* Efeitos calculados a partir do valor da constante de velocidade 
meia-vida), calculados de acordo com expressões que correspondem a cinéticas de pseudoprimeira ordem. ${ }^{17}$

Analisando-se os valores dos efeitos (Tabela 1), calculados a partir do valor da constante de velocidade, é possível observar que o aumento da concentração de peróxido e de íon ferroso promove um significativo aumento no valor da constante de velocidade. Uma vez que ambos os valores são superiores ao valor do desvio calculado a partir da triplicata do ponto central $(0,005)$, é possível admitir que ambos os parâmetros apresentem um efeito significativo, o qual indica a conveniência de se utilizar maiores concentrações de peróxido e maiores concentrações de íon ferroso. Nestas condições (experimento 4) se observam o maior valor de constante de velocidade $(0,422)$ e o menor valor de tempo de meia-vida $(1,64)$.

Experiências prévias demonstram que as referidas concentrações permitem uma eficiência máxima de degradação, em função de maiores concentrações, tanto de $\mathrm{Fe}^{2+}$ como de $\mathrm{H}_{2} \mathrm{O}_{2}$, exercerem efeito sequestrante de radical hidroxila, o que tende a diminuir a eficiência do processo. Por outro lado, a experiência também indica que, mantendo-se a concentração dos substratos na mesma ordem de grandeza, a degradação se processa de forma similar, não sendo significativamente influenciada pelas diferenças estruturais apresentadas pelos corantes modelo. Desta forma, optou-se por selecionar a condição do experimento 4 ( $150 \mathrm{mg} \mathrm{L}^{-1} \mathrm{de}_{2} \mathrm{O}_{2}$ e $15 \mathrm{mg} \mathrm{L}^{-1} \mathrm{de} \mathrm{Fe}^{2+}$ ) para a realização dos estudos subsequentes.

Nestas condições, a descoloração do corante modelo se processa rapidamente (Figura 1), o que permite a eliminação praticamente completa da cor em 5 min de tratamento. Mudanças significativas também são observadas na região ultravioleta do espectro, mudanças que, entretanto, são principalmente associadas à presença de peróxido de hidrogênio, que absorve fortemente na região compreendida entre 200 e 300 nm. Em função desta interferência, uma interpretação mais aprofundada destas mudanças, principalmente no que diz respeito à degradação das formas aromáticas do corante, é praticamente impossível.

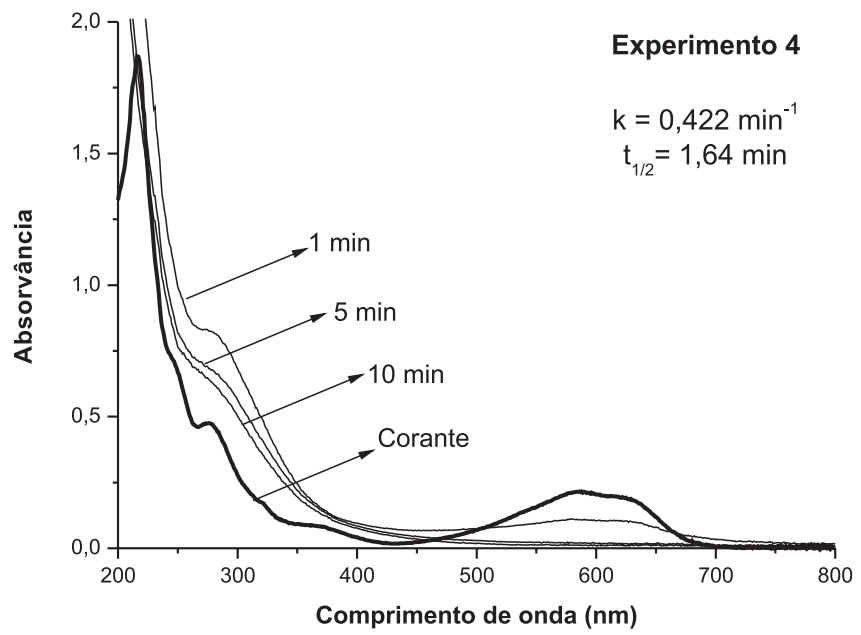

Figura 1. Modificação do espectro UV-Vis durante o tratamento da solução de corante azul QR 19 por processo Fenton (experimento 4)

Resultados similares foram relatados por Chang e colaboradores, ${ }^{23}$ em estudos de degradação do corante Azul QR 19 por processos Fenton.

Muitos autores consideram que a reação de Fenton pode efetivamente oxidar e transformar moléculas orgânicas, sem, no entanto, mineralizar completamente as estruturas que surgem no processo. Gutowska e colaboradores, ${ }^{24}$ por exemplo, estudando a degradação de um corante azo (Laranja Reativo 113) verificaram a oxidação parcial do substrato, com formação de fragmentos aromáticos substituídos, os quais, em tratamentos prolongados, podem ser convertidos em ácidos carboxílicos, aldeídos e alcoóis. Mineralização parcial e formação de intermediários de natureza aromática também foram relatadas por Fan e colaboradores ${ }^{25}$ em estudos de degradação envolvendo o corante violeta cristal e processos Fenton.

A variação da concentração de peróxido de hidrogênio e a evolução de formas ferrosas e férricas foram monitoradas durante o processo de degradação, encontrando-se os resultados apresentados na Figura 2. Estes resultados permitem ilustrar um dos principais inconvenientes do processo Fenton aplicado de forma convencional. Isto é, embora o peróxido de hidrogênio seja consumido lentamente (Figura 2), o que implica em concentrações residuais elevadas até em tempos de reação da ordem de $60 \mathrm{~min}$, a cinética da reação se torna desfavorável a partir de 30 min de reação, em função da baixa concentração residual de íons ferrosos (aproximadamente $3 \mathrm{mg} \mathrm{L}^{-1}$ ) e da menor capacidade catalítica das formas férricas produzidas. Deste ponto de vista, a utilização de processos assistidos por radiação pode se tornar vantajosa, em razão da fotorredução de íons férricos permitir a regeneração das formas ferrosas, mais ativas.

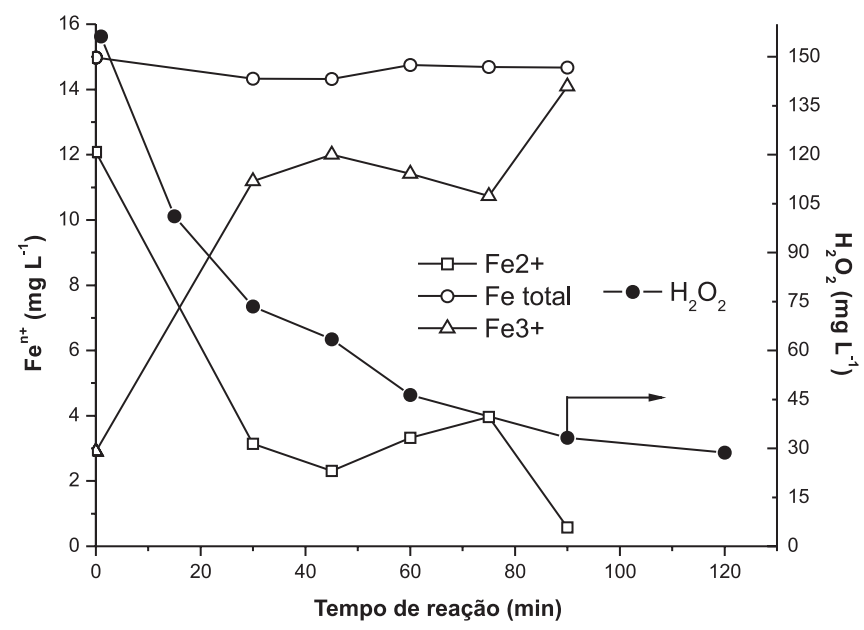

Figura 2. Consumo de $\mathrm{H}_{2} \mathrm{O}_{2}$ e evolução de espécies ferrosas e férricas durante a degradação do corante azul QR 19 por processo Fenton

Estudos de degradação também foram realizados com um segundo corante modelo, Alaranjado Reativo 16, utilizando-se as mesmas condições operacionais do estudo anterior, confirmando-se a rápida descoloração do substrato modelo, o que implicou em eficiente degradação do grupo cromóforo e redução praticamente completa do sinal espectral registrado entre 450 e $550 \mathrm{~nm}$. Assim como demonstrado nos estudos de degradação do corante Azul QR-19, a região entre 200 e $300 \mathrm{~nm}$ sofre significativa influência das altas concentrações iniciais de peróxido de hidrogênio. Desta forma, modificações do perfil espectral nesta região não são significativas, sendo impossível, no intervalo de tempo em que a reação foi processada (60 min), confirmar a degradação de estruturas aromáticas e a consequente mineralização do substrato.

\section{Degradação de corantes modelo pelo sistema foto-Fenton}

Trabalhando-se nas mesmas condições utilizadas no processo Fenton (200 mL de solução de corante, concentração de $20 \mathrm{mg} \mathrm{L}^{-1}$, $\mathrm{pH} 3,15 \mathrm{mg} \mathrm{L}^{-1}$ de $\mathrm{Fe}^{2+}$ e $150 \mathrm{mg} \mathrm{L}^{-1} \mathrm{de}_{2} \mathrm{O}_{2}$ ), foi avaliada a degradação dos corantes modelo por processos foto-Fenton assistidos por radiação UV-A (320-400 nm), protegendo-se a lâmpada a vapor de mercúrio com um bulbo de vidro Pyrex.

Nestas condições, o monitoramento espectrofotométrico do processo de degradação do corante Azul QR 19 demonstra uma elevada velocidade de reação, o que permite a completa remoção da cor em 
tempos de reação da ordem de 1 min (Figura 3). De maneira geral, antecedentes da literatura mostram que a degradação de moléculas deste tipo por processos avançados implica uma rápida quebra da estrutura dos grupos cromóforos, com formação de derivados que mantêm a sua aromaticidade. Posteriormente, pequenos fragmentos de caráter fenólico são formados, os quais, finalmente, dão lugar a moléculas de ácidos carboxílicos de cadeia curta, tipicamente ácidos oxálico e acético. ${ }^{26} \mathrm{~A}$ modificação do sinal espectral observado na região ultravioleta confirma esta hipótese, uma vez que a destruição do cromóforo, associada ao processo de descoloração, se dá de maneira muito mais rápida do que a diminuição do sinal na região centrada entre 200 e $300 \mathrm{~nm}$. De qualquer forma, a região que caracteriza absorção por parte de espécies aromáticas diminui significativamente a sua intensidade em tempos de reação da ordem de $10 \mathrm{~min}$, o que atesta graus avançados de degradação.

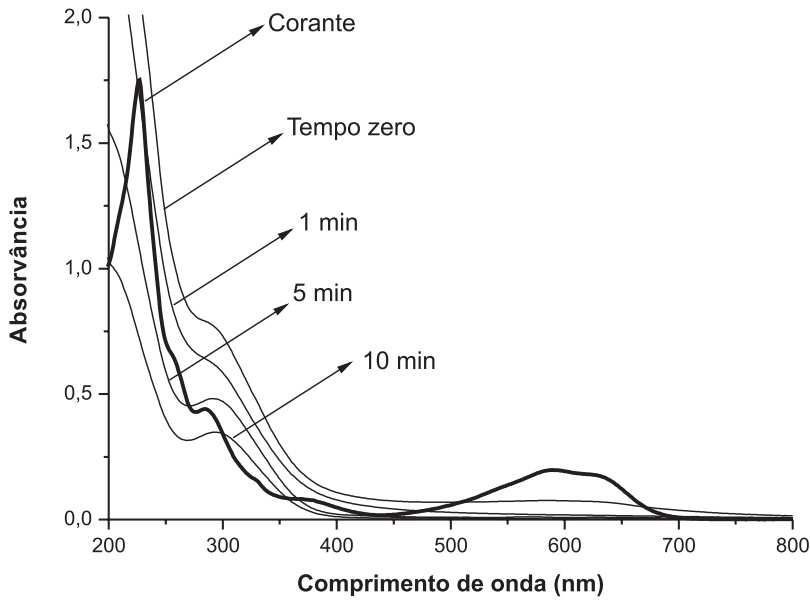

Figura 3. Modificação do espectro UV-Vis durante o tratamento da solução de corante azul QR 19 por processo foto-Fenton

Adicionalmente, observa-se que a eficiência de degradação do processo foto-Fenton é superior à apresentada pelo processo Fenton, basicamente em função da radiação permitir a regeneração de íon ferroso por fotorredução do íon férrico e a consequente geração de mais um equivalente de radical hidroxila. Entretanto, embora as espécies ferrosas possam ser mantidas em elevada concentração no decorrer do processo fotoassistido, a reação se torna desfavorável a partir dos primeiros 15 min de tratamento, em razão do rápido consumo de peróxido de hidrogênio e da consequente baixa concentração residual (Figura 4). Deste ponto de vista, a contínua reposição de $\mathrm{H}_{2} \mathrm{O}_{2}$ pode implicar em significativas melhoras na capacidade de degradação do sistema foto-Fenton, o que corresponde à principal proposta dos sistemas eletro-Fenton.

O processo foto-Fenton também foi aplicado em estudos de degradação do corante Laranja Reativo 16, nas mesmas condições operacionais do experimento anterior. Os resultados confirmam a rápida degradação do grupo cromóforo, o que permite completa remoção da cor em tratamentos da ordem de $10 \mathrm{~min}$. Da mesma forma que ocorre na degradação do corante Azul QR-19, verifica-se um sensível aumento preliminar do sinal na região ultravioleta (200-300 nm), por conta da presença de elevadas concentrações de peróxido de hidrogênio. Posteriormente, o sinal é sistematicamente reduzido, o que, além de indicar consumo de peróxido, implica em degradação de estruturas aromáticas transientes.

\section{Degradação de corantes modelos pelo sistema eletro-Fenton}

Inicialmente, inúmeros testes foram realizados com a finalidade

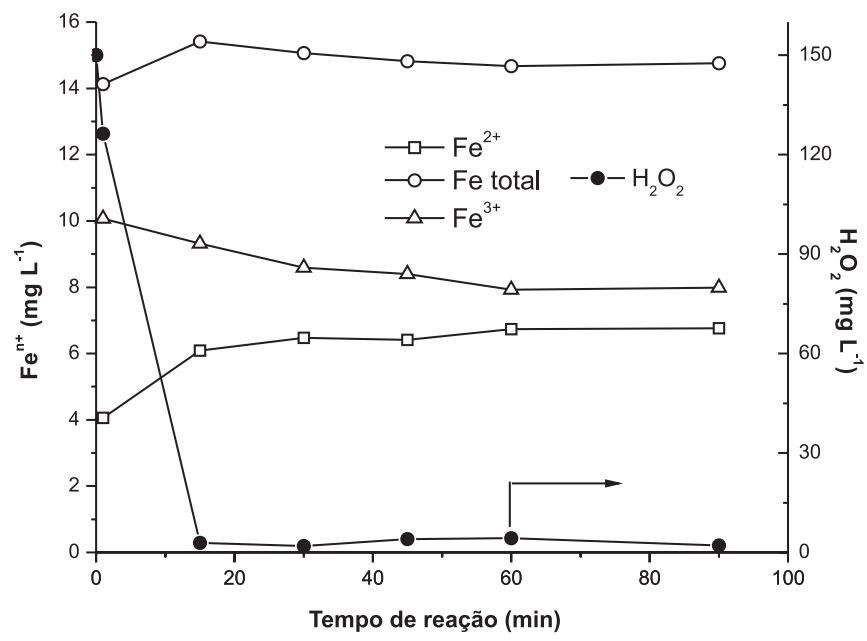

Figura 4. Consumo de $\mathrm{H}_{2} \mathrm{O}_{2}$ e evolução de espécies ferrosas e férricas durante a degradação do corante azul QR 19 por processo foto-Fenton

de identificar o melhor conjunto de materiais catódicos e anódicos, objetivando maximizar a geração eletroquímica de peróxido de hidrogênio. Os experimentos envolveram a combinação de diferentes materiais eletródicos na forma de placas e o monitoramento da produção de peróxido, utilizando-se condições previamente definidas em função de antecedentes da literatura (eletrólito: $\mathrm{Na}_{2} \mathrm{SO}_{4} 0,05 \mathrm{~mol} \mathrm{~L}{ }^{-1}$, densidade de corrente: $17 \mathrm{~mA} \mathrm{~cm}^{-2}$, soluções saturadas em oxigênio). ${ }^{27}$

Os resultados, resumidamente apresentados na Tabela 2, indicam uma maior eficiência do sistema carbono-felt (cátodo) e aço inox (ânodo), com uma produção de aproximadamente $19 \mathrm{mg} \mathrm{L}^{-1}$ de peróxido em 60 min de reação. Infelizmente, estudos posteriores realizados na presença de ânodos de aço inox evidenciaram importante corrosão do eletrodo, com a consequente liberação de $\mathrm{Cr}^{3+} \mathrm{em}$ solução. Por este motivo, foram realizados estudos subsequentes utilizando-se o par CF-Pt.

Tabela 2. Efeito dos materiais eletródicos na produção eletroquímica de $\mathrm{H}_{2} \mathrm{O}_{2}$ (Tempo de reação: $60 \mathrm{~min}$ )

\begin{tabular}{|c|c|c|}
\hline Cátodo & Ânodo & $\mathrm{H}_{2} \mathrm{O}_{2}\left(\mathrm{mg} \mathrm{L}^{-1}\right)$ \\
\hline Carbono-felt & $\mathrm{Pt}$ & 9,0 \\
\hline Carbono-felt & $\mathrm{Ti} / \mathrm{TiO}_{2} \mathrm{RuO}_{2}$ & 7,4 \\
\hline Carbono-felt & Aço Inox & 19,3 \\
\hline $\mathrm{Ti} / \mathrm{TiO}_{2} \mathrm{RuO}_{2}$ & $\mathrm{Ti} / \mathrm{TiO}_{2} \mathrm{RuO}_{2}$ & $<\mathrm{LQ}^{*}$ \\
\hline $\mathrm{Ti} / \mathrm{TiO}_{2} \mathrm{RuO}_{3}$ & $\mathrm{Pt}$ & 8,5 \\
\hline $\mathrm{Ti} / \mathrm{TiO}_{2} \mathrm{RuO}_{4}$ & Aço Inox & $<\mathrm{LQ}$ \\
\hline Aço Inox & Aço Inox & $<\mathrm{LQ}$ \\
\hline Aço Inox & $\mathrm{Pt}$ & $<\mathrm{LQ}$ \\
\hline Aço Inox & $\mathrm{Ti} / \mathrm{TiO}_{2} \mathrm{RuO}_{2}$ & $<\mathrm{LQ}$ \\
\hline
\end{tabular}

$* \mathrm{LQ}=2,5 \mathrm{mg} \mathrm{L}^{-1}$

Muitos autores referenciam que a utilização de ânodos de Pt e cátodos de carbono-felt permite uma elevada eficiência nos processos eletro-Fenton, em função do sistema proporcionar uma maior capacidade de regeneração do íon ferroso. No entanto, a construção desses sistemas eletroquímicos de degradação geralmente necessita de peças de Pt de elevada área superficial, o que aumenta o custo do tratamento. Nas condições em que este estudo foi realizado foi possível observar uma eficiente produção de $\mathrm{H}_{2} \mathrm{O}_{2}$ com o sistema CF-Pt, principalmente levando-se em consideração o pequeno tamanho dos ânodos utilizados $\left(6 \mathrm{~cm}^{2}\right)$. 
Após os estudos preliminares, realizados com eletrodos em formato de placas de $21 \mathrm{~cm}^{2}$, foi construído um eletrodo cilíndrico de carbono-felt com área superficial resultante de $253 \mathrm{~cm}^{2}$ (Figura 1S, material suplementar). Primeiramente, foi utilizado um cilindro de PVC perfurado $(\mathrm{h}=11 \mathrm{~cm}, \mathrm{~d}=4,5 \mathrm{~cm})$ para funcionar como suporte para o material catódico. Foi utilizada uma membrana separadora de baterias de $\mathrm{Pb}$ (250 $\mathrm{g} \mathrm{m}^{-2}$, gentilmente cedida pelo LACTEC) envolvendo o cilindro de PVC, para que o material catódico não permanecesse em contato direto com o cilindro (Figura 1SA, material suplementar). Em seguida, uma peça de carbono-felt (11 x 23 x 1,0 $\mathrm{cm}$ ) foi fixada no suporte com o auxílio de abraçadeiras de nylon, juntamente com duas lâminas de aço inox que fazem o contato elétrico (Figura 1SB, material suplementar). Após a confecção do eletrodo, o sistema eletroquímico pode ser montado de maneira que o ânodo permanece-se inserido na cavidade central do cátodo (Figuras 1SC e $1 \mathrm{SD}$, material suplementar).

Inicialmente, a geração eletroquímica de $\mathrm{H}_{2} \mathrm{O}_{2}$ foi avaliada utilizando-se diversas condições de densidade de corrente (J: 0,4 a $\left.2,0 \mathrm{~mA} \mathrm{~cm}{ }^{-2}\right)$ e de concentração de eletrólito $\left(\mathrm{Na}_{2} \mathrm{SO}_{4}: 0,025\right.$ a 0,075 mol L $\mathrm{L}^{-1}$ ), sempre em pH 3 e em sistema oxigenado. Os resultados, resumidamente apresentados na Figura 5, indicam que a maior eficiência na produção de $\mathrm{H}_{2} \mathrm{O}_{2}$ pode ser alcançada trabalhando-se com a maior concentração de eletrólito $\left(0,075 \mathrm{~mol} \mathrm{~L}^{-1}\right)$ e uma densidade de corrente aplicada de $1,6 \mathrm{~mA} \mathrm{~cm}{ }^{-2}$. Nestas condições $63 \mathrm{mg} \mathrm{L}^{-1}$ de $\mathrm{H}_{2} \mathrm{O}_{2}$ são acumulados em 60 min de reação, concentração que se mostra superior à reportada por Wang, ${ }^{27} \mathrm{em}$ estudos envolvendo a degradação de corantes por processos eletro-Fenton.

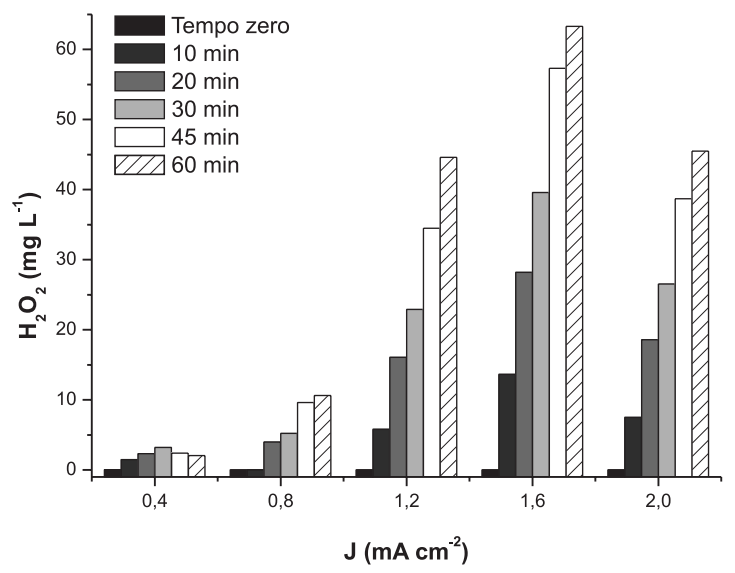

Figura 5. Concentração de $\mathrm{H}_{2} \mathrm{O}_{2}$ eletroquimicamente gerada utilizando-se solução de $\mathrm{Na}_{2} \mathrm{SO}_{4} 0,075 \mathrm{~mol} \mathrm{~L}^{-1}$, em função da densidade de corrente (J)

Após a etapa de configuração e otimização do sistema eletroquímico, foram realizados estudos de degradação com soluções aquosas do corante modelo (Azul QR-19, $1000 \mathrm{~mL}, 20 \mathrm{mg} \mathrm{L}^{-1}$ ), por processos eletro-Fenton. Todos os ensaios foram realizados nas condições de maior eficiência na produção de $\mathrm{H}_{2} \mathrm{O}_{2}$ (cátodo de $\mathrm{CF}$ de $253 \mathrm{~cm}^{2}$, ânodo de Pt de $6,0 \mathrm{~cm}^{2}$, densidade de corrente de $1,6 \mathrm{~mA} \mathrm{~cm} \mathrm{~cm}^{-2} \mathrm{e}$ oxigenação contínua), propiciando-se a reação de Fenton pela adição de $\mathrm{Fe}^{2+}$ em concentração de $15 \mathrm{mg} \mathrm{L}^{-1}$.

A partir da sequência de espectros apresentada na Figura 6 é possível observar uma rápida degradação do cromóforo, o que permite uma descoloração bastante avançada do corante em tempos de reação da ordem de 20 min. Levando-se em consideração apenas o processo de descoloração, observa-se que o processo Fenton, aplicado em meio homogêneo convencional (Figura 1), provoca uma descoloração mais rápida do corante modelo, obviamente em função da maior concentração inicial de $\mathrm{H}_{2} \mathrm{O}_{2}\left(150 \mathrm{mg} \mathrm{L}^{-1}\right)$. Entretanto, diferenças que tornam favorável o processo eletro-Fenton podem ser observadas em maiores tempos de reação. Isto é, enquanto o processo Fenton convencional se processa lentamente na presença de elevadas concentrações de $\mathrm{H}_{2} \mathrm{O}_{2}$ residual e baixas concentrações de $\mathrm{Fe}^{2+}$, o processo eletro-Fenton se sustenta por mais tempo, permitindo uma significativa redução na área espectral, o que, de maneira geral, se correlaciona muito bem com a remoção da carga orgânica no sistema.

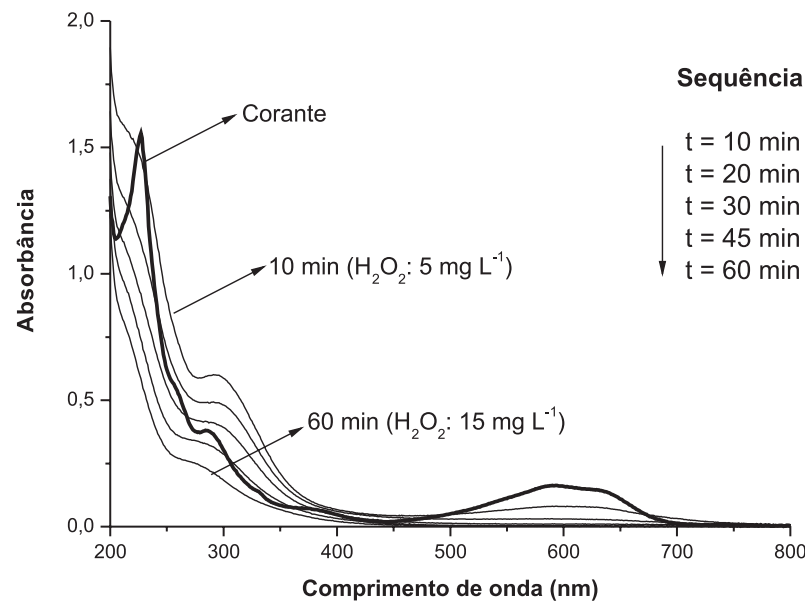

Figura 6. Modificação do espectro UV-Vis durante o tratamento da solução de corante azul QR 19 por processo eletro-Fenton

Resultados igualmente promissores foram observados nos estudos de degradação envolvendo o corante Laranja Reativo 16.

Nas condições em que o processo foi aplicado, foram observadas concentrações crescentes de peróxido residual, o que permite supor a possibilidade de se estender a reação de Fenton por um tempo significativamente maior ao permitido pelo processo Fenton convencional. Entretanto, a evolução de formas ferrosas e férricas continua sendo desfavorável, principalmente em razão da rápida conversão verificada nos primeiro minutos de reação. Levando-se em consideração que a conversão de espécies férricas em ferrosas pode ser viabilizada pela componente eletroquímica, a procura de condições que permitam esta conversão se apresenta extremamente importante, fazendo parte de estudos futuros.

De qualquer forma, o conjunto de resultados apresentado anteriormente confirma o adequado funcionamento da célula eletrolítica para produção in situ de $\mathrm{H}_{2} \mathrm{O}_{2}$, assim como a sua utilidade em processos de degradação por processos Fenton.

\section{Estudo de degradação de resíduos de tingimento}

Os estudos de remediação de efluentes têxteis envolveram o uso de resíduos aquosos gentilmente cedidos por uma empresa de tinturaria localizada na região de Curitiba. Proveniente de operações de tingimento de fibras de algodão, o resíduo apresenta $\mathrm{pH}$ 6, forte coloração azul e presença de material particulado.

Primeiramente, as partículas presentes no resíduo foram retiradas por filtração e o pH ajustado em 3,0. O processo eletro-Fenton foi aplicado seguindo-se as mesmas condições utilizadas na degradação dos substratos modelos $\left(0,075 \mathrm{~mol} \mathrm{~L}^{-1} \mathrm{de} \mathrm{Na}_{2} \mathrm{SO}_{4}, 15 \mathrm{mg} \mathrm{L}^{-1} \mathrm{de} \mathrm{Fe}^{2+}\right.$, sistema oxigenado, densidade de corrente de $1,6 \mathrm{~mA} \mathrm{~cm}^{-2}$, cátodo de $\mathrm{CF}$ de $253 \mathrm{~cm}^{2}$ e ânodo de Pt de $6,0 \mathrm{~cm}^{2}$ ), utilizando $1000 \mathrm{~mL}$ do resíduo.

De acordo com o monitoramento espectrofotométrico (Figura 7) a cor do resíduo é lentamente removida, alcançando valores residuais da ordem de $10 \%$ em tratamentos de $45 \mathrm{~min}$. Isto indica que a quebra do grupo cromóforo se processa de maneira mais vagarosa, em comparação aos processos Fenton e foto-Fenton tradicionais, 
principalmente em razão das elevadas concentrações iniciais de peróxido de hidrogênio utilizadas nestes processos $\left(150 \mathrm{mg} \mathrm{L}^{-1}\right)$. Na região ultravioleta se observa um significativo aumento de sinal (200-300 nm) nos primeiros minutos de tratamento, em decorrência da presença de peróxido de hidrogênio eletrogerado, que também absorve fortemente nesta região. Após 30 min de reação é possível observar que o sinal começa a decair, sugerindo consumo de peróxido e degradação eficiente das estruturas aromáticas que caracterizam a mistura de corantes presentes no resíduo.

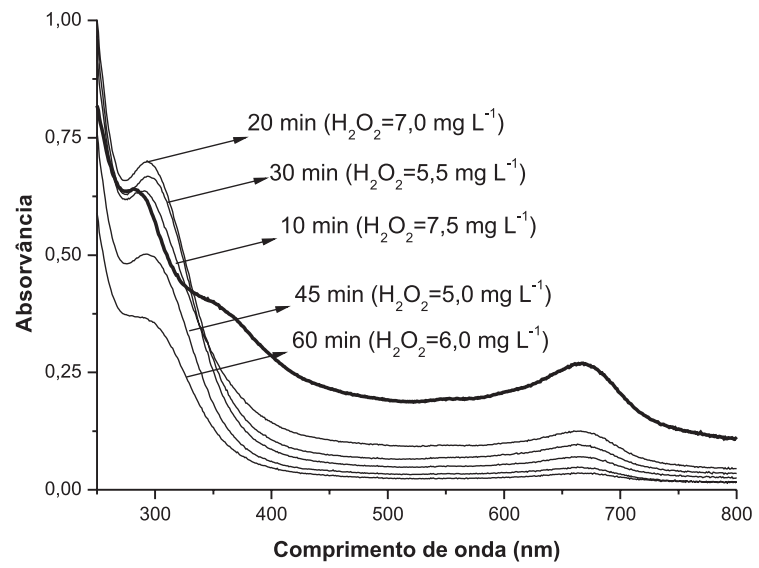

Figura 7. Modificação do espectro UV-Vis durante o tratamento do resíduo do tingimento por processo eletro-Fenton

Como o peróxido produzido é rapidamente consumido através da reação Fenton convencional, não é possível verificar altas concentrações do produto durante a reação eletro-Fenton. Entretanto, observa-se que em 60 min de tratamento a concentração de $\mathrm{H}_{2} \mathrm{O}_{2}$ aumenta ligeiramente, provavelmente em função da baixa concentração de íons ferrosos, que se apresenta em valores não detectáveis a partir de 45 min de reação.

Embora muitos trabalhos tenham demonstrado a elevada eficiência de degradação dos processos eletro-Fenton frente a soluções aquosas de corantes têxteis, poucos são os relatos de aplicação no tratamento de resíduos industriais. Dentro deste contexto, pode ser dado destaque a uma publicação recente, ${ }^{15}$ que demonstra elevada eficiência do processo no tratamento de resíduos aquosos contendo corantes reativos do tipo azo.

Finalmente, é importante salientar que, em função da sua complexa natureza radicalar, os processos Fenton podem levar à geração de espécies transientes de maior toxicidade do que o composto de partida, o que é particularmente importante no tratamento de corantes têxteis, os quais usualmente não apresentam toxicidade aguda de significância. Para avaliar a expressão de toxicidade aguda, foram realizados estudos utilizando-se vários organismos teste (ex. Artemia salina e Selenastrum capricornutum), não se observando toxicidade em nenhum tempo monitorado (resultados não apresentados).

\section{CONCLUSÕES}

Nos estudos preliminares, envolvendo a degradação de corantes modelo (Azul QR-19 e Laranja Reativo 16) ficou demonstrada a elevada capacidade de degradação dos processos Fenton e foto-Fenton. Aplicados em condições experimentais otimizadas ( $\mathrm{pH}: 3$; $\mathrm{H}_{2} \mathrm{O}_{2}: 150 \mathrm{mg} \mathrm{L}^{-1} ; \mathrm{Fe}^{2+}: 15 \mathrm{mg} \mathrm{L}^{-1}$ ), ambos os processos permitem a rápida degradação dos grupos cromóforos, acompanhada de remoção praticamente completa da cor em tempos de reação da ordem de 15 min. De maneira geral, a presença de elevadas quantidades de peróxido de hidrogênio em solução impede uma adequada visualização das modificações espectrais na região ultravioleta. Entretanto, nos processos assistidos por radiação, em que o consumo de peróxido se dá mais rapidamente, é possível verificar ausência de sinais na região compreendida entre 200 e 300 nm, o que implica em completa degradação dos intermediários aromáticos característicos. Embora a ausência de sinais nesta região implique em graus avançados de degradação, a mineralização completa não pode ser assegurada, em função do acúmulo frequente de ácidos carboxílicos no final da reação.

Nos processos Fenton, o lento consumo de $\mathrm{H}_{2} \mathrm{O}_{2}$ poderia garantir a ocorrência de reações por tempo de até 60 min. Entretanto, o rápido consumo de $\mathrm{Fe}^{2+}$ inviabiliza a reação por tempos prolongados, o que representa uma das maiores limitações do processo. Por sua vez, a presença de radiação poderia estender o tempo de aplicação do processo foto-Fenton, em virtude da regeneração contínua de espécies ferrosas. Infelizmente, a presença de radiação induz um rápido consumo de peróxido, o que torna o processo de degradação desfavorável a partir de tempos de reação da ordem de $20 \mathrm{~min}$. Deste ponto de vista, a contínua geração de peróxido representa uma boa alternativa para se aumentar o tempo de ação dos processos Fenton, o que justifica o desenvolvimento de processos eletro-Fenton.

O sistema eletroquímico de trabalho foi configurado com um cátodo cilíndrico de carbono-felt com área superficial de $253 \mathrm{~cm}^{2}$, juntamente com um ânodo de Pt de $6,0 \mathrm{~cm}^{2}$. Nestas condições, a geração de $\mathrm{H}_{2} \mathrm{O}_{2}$ foi maximizada $\left(63 \mathrm{mg} \mathrm{L}^{-1}\right)$ trabalhando-se em meio oxigenado $(1000 \mathrm{~mL})$, com aplicação de densidade de corrente de $1,6 \mathrm{~mA} \mathrm{~cm}{ }^{-2}$ e concentração de eletrólito $\left(\mathrm{Na}_{2} \mathrm{SO}_{4}\right)$ de $0,075 \mathrm{~mol}$ $\mathrm{L}^{-1}$. Nos estudos de degradação de corantes modelo foi constatada degradação eficiente dos grupos cromóforos, com remoção completa da cor em tempos de reação da ordem de 30 min. Embora o processo de degradação se dê de forma mais lenta, em relação aos processos Fenton convencionais, o processo eletro-Fenton se sustenta por mais tempo, graças à contínua geração de peróxido.

\section{MATERIAL SUPLEMENTAR}

Está disponível em http://quimicanova.sbq.org.br, na forma de arquivo pdf, com acesso livre. Na Figura $1 \mathrm{~S}$ apresentam-se alguns detalhes do sistema utilizado nos estudos de degradação por processos eletro-Fenton.

\section{AGRADECIMENTOS}

Ao Conselho Nacional de Desenvolvimento Científico e Tecnológico $(\mathrm{CNPq})$ pelo suporte financeiro ao projeto (Processo No 553673/2009-2). Os autores agradecem especialmente a gentileza do Prof. Dr. A. Carubelli (in memoriam) que, de muitas maneiras, contribuiu com a realização deste trabalho.

\section{REFERÊNCIAS}

1. Khouni, I.; Marrot, B.; Moulin, P.; Amar, R. B.; Desalination 2011, 268, 27.

2. Zahrim, A. Y.; Tizaoui, C.; Hilal, N.; Desalination 2011, 266, 1.

3. Chuah, T. G.; Jumasiah, A.; Azni, I.; Katayon, S.; Thomas Choong, S. Y.; Desalination 2005, 175, 305.

4. Chaari, I.; Feki, M.; Medhioub, M.; Bouzid, J.; Fakhfakh, E.; Jamoussi, F.; J. Hazard. Mater. 2009, 172, 1623.

5. Saratale, R. G.; Saratale, G. D.; Chang, J. S.; Govindwar, S. P.; J. Taiwan Inst. Chem. Eng. 2011, 42, 138.

6. Couto, S. R.; Biotechnol. Adv. 2009, 27, 227.

7. Pirillo, S.; García Einschlag, F. S.; Ferreira, M. L.; Rueda, E. H.; J. Mol. Catal. B: Enzym. 2010, 66, 63. 
8. Han, F.; Kambala, V. S. R.; Srinivasan, M.; Rajarathnam, D.; Naidu, R.; Appl. Catal., A 2009, 359, 25.

9. Chang, M.; Chern, J.; J. Taiwan Inst. Chem. Eng. 2010, 41, 221.

10. Rauf, M. A.; Salman Ashraf, S.; Chem. Eng. J. 2009, 151, 1.

11. Núñez, L.; García-Hortal, J. A.; Torrades, F.; Dyes Pigm. 2007, 75, 647.

12. Neyens, E.; Baeyens, J.; J. Hazard. Mater. 2003, 98, 33.

13. Nogueira, R. F. P.; Trovó, A. G.; Silva, M. R. A.; Villa, R. D.; Oliveira, M. C.; Quim. Nova 2007, 30, 400.

14. Rosales, E.; Pazos, M.; Longo, M. A.; Sanromán, M. A.; Chem. Eng. J. 2009, 155, 62.

15. El-Desoky, H. S.; Ghoneim, M. M.; El-Sheikh, R.; Zidan, N. M.; J. Hazard. Mater. 2010, 175, 858

16. Panizza, M.; Cerisola, G.; Water Res. 2009, 43, 339.

17. Özcan, A.; Oturan, M. A.; Oturan, N.; Şahin, Y.; J. Hazard. Mater. 2009, $163,1213$.

18. Özcan, A.; Şahin, Y.; Oturan, M. A.; Chemosphere 2008, 73, 737.

19. Isarain-Chávez, E.; Arias, C.; Cabot, P. L.; Centellas, F.; Rodríguez, R. M.; Garrido, J. A.; Brillas, E.; Appl. Catal. B: Environ. 2010, 96, 361.
20. Pimentel, M.; Oturan, N.; Dezotti, M.; Oturan, M. A.; Appl. Catal. B: Environ. 2008, 83, 140.

21. Silva, M. R. A.; Oliveira, M. C.; Nogueira, R. F. P.; Eclética Química 2004, 29, 19.

22. APHA; Standart Methods for the Examination of Water and Wastewater, $19^{\text {th }}$ ed., WPCF: New York, 1995, p. 5220.

23. Chang,S.; Chuang, S.; Li, H.; Liang, H.; Huang, L.; J. Hazard. Mater. 2009, 166, 1279.

24. Gutowska, A.; Kałużna-Czaplińska, J.; Józwiak, W. K.; Dyes Pigm. 2007, 74, 41.

25. Fan, H.; Huang, S.; Chung, W.; Jan, J.; Lin, W.; Chen, C.; J. Hazard. Mater. 2009, 171, 1032.

26. Gözmen, B.; Kayan, B.; Gizir, A. M.; Hesenov, A.; J. Hazard. Mater. 2009, 168, 129.

27. Wang, A.; Qu, J.; Ru, J.; Liu, H.; Ge, J.; Dyes Pigm. 2005, 65, 227. 